\title{
Examination of an Oscillating Flame in the Turbulent Flow around a Bluff Body with Large Eddy Simulation based on the Probability Density Function Method
}

\author{
W. P. Jones · M. Jurisch · A. J. Marquis
}

Received: date / Accepted: date

\begin{abstract}
The present paper describes a Large Eddy Simulation modelling framework for the simulation of oscillating flames in practical flow configurations. The unresolved subfilter scale motion is modelled using the dynamic Smagorinsky model in combination with the Probability Density Function method. It is shown that the Large Eddy Simulation method is capable of reproducing the characteristic shape of the reaction zone as well as the nonlinear evolution of the total heat release rate in a bluff-body stabilised combustor. Commonly used measures for quantifying the variation of the total heat release rate are evaluated and examined in the present flow configuration of a lean-premixed ethylene-air flame. It was found that formaldehyde-based measures do not appropriately reproduce the amplitude and phase of the total heat release rate. A significantly improved correlation was achieved by employing the product of the mass fractions of molecular oxygen $\left(\mathrm{O}_{2}\right)$ and the ketenyl radical $(\mathrm{HCCO})$ as a means of characterising the variation of the total heat release rate.
\end{abstract}

Keywords flame oscillation $\cdot$ heat release rate $\cdot$ gas turbine combustors

\section{Introduction}

Manufacturers of stationary gas turbine combustors are challenged to develop efficient and low-emission combustion devices in order to comply with legislation to reduce carbon emissions. One of the major problems which needs to be addressed in the design process is flame oscillation. In the worst case scenario it can lead to structural damage or even failure of the entire combustion system. Flame oscillation is characterised by quasi-periodic largeamplitude variations of the fields describing the motion of the mixture, such as temperature, pressure, and velocity, and must clearly be distinguished from irregular fluctuations with comparatively low-amplitude induced by turbulence. It appears as the result of a complex interaction between the zone in which the chemical reactions proceed (reaction zone), and the fluid motion in the neighbourhood of the reaction zone. Theory, experiments and simulations have revealed that the dynamics of oscillating flames are highly sensitive to the

W. P. Jones · M. Jurisch · A. J. Marquis

Department of Mechanical Engineering, Imperial College London, Exhibition Road, London, SW7 2AZ, UK E-mail: w.jones@imperial.ac.uk 
boundary conditions whether associated with the velocity, mixture composition and temperature at the injection locations or the type of fuel and oxidiser, to name only a few. There is a large diversity in the possible mechanisms which can trigger flame oscillation, but a general discussion of these lies outside the scope of this paper. The interested reader may consult Matalon [25].

A well-suited method for the quantitative prediction of the dynamics of reacting mixtures in the context of turbulent fluid motion is Large Eddy Simulation (LES). LES involves a description of the fluid motion on length scales which lie above an a priori defined threshold associated, for example, with the local resolution of the computational grid or based on an estimated physical length scale- the 'resolved' motion. This is achieved by the application of a spatial filter, with width $\Delta$, to the equations of motion; the threshold is generally referred to as the filter scale. The unresolved fluid motion at and below the sub-filter scale is modelled and with an appropriately chosen filter scale makes only a minor contribution to the total turbulent kinetic energy: the explicitly resolved fluid motion carries with it the greater fraction of the total turbulent kinetic energy. Given that the large scale and energetic motions are largely responsible for turbulent transport, LES thus maintains good accuracy whilst being computationally cost effective. One of the main challenges in achieving an accurate representation of turbulent flames with LES lies in the modelling of the filtered net chemical species formation rates, arising through chemical reaction, which appear in the conservation equations for the mass fractions of the constituents of the mixture. The filtered formation rates are strongly affected by the unresolved sub-filter scale fluctuations of the species mass fractions and temperature. These can be accounted for by the introduction of a transport equation for the filtered one-point, one-time joint Probability Density Function (PDF) for all of the scalar quantities required to describe chemical reaction. The PDF method does not involve the assumption of any particular combustion regime and therefore offers a wide range of applicability, including non-premixed, and premixed, as well as stratified and spray combustion, see for example Bulat et al. [7] and Jones et al. [19,21,22]. Thus far the method has not been assessed in the context of oscillating flames. There are no conceptual difficulties in incorporating detailed reaction mechanisms into the PDF method which is particularly important in the modelling of unsteady chemical reactions. An accurate prediction of the heat release rate involves typically 20 or more chemical species and is essential in the study of flame-turbulence interaction and flame oscillation.

In the present paper, the application of LES with a PDF sub-filter scale model to the study of flame oscillation in the turbulent flow around a conical bluff body is reported and the results compared with measurements. Commonly used measures of the total heat release rate in the investigated flow configuration are then examined. Previous simulations of this same flow have focussed on the response of an inert air flow to acoustic perturbation [3], the prediction of the Flame Transfer Function (FTF) [2], and the ignition characteristics [29]. The remaining part of the paper is organised as follows. In Section 2 details of the LES method are provided. This is followed by a brief description of the underlying experiment and an outline of the applied modelling framework in Sections 3 and 4, respectively. In Section 5 and 6 the results are discussed and the findings summarised. 


\section{Large Eddy Simulation}

\subsection{Filtered field equations}

The scale separation in LES is achieved by applying a spatial filter to the field equations describing the motion of the mixture. In the description of reacting mixtures it is common practice to use a density-weighted filter due to large variations of the density arising flames. The field equations in the present case consist of the Continuity equation, the Navier-Stokes equation and the conservation equations for mass fractions of $N$ chemical species and the energy of the mixture. The mixture is here assumed to be incompressible which implies that thermo-acoustic effects cannot be accounted for within the present framework. However thermo-acoustic effects do not exert an important influence in the case to be considered. Using standard notation the filtered field equations can be cast into the following form

Continuity

$$
\frac{\partial \bar{\rho}}{\partial t}+\frac{\partial \bar{\rho} \widetilde{u}_{i}}{\partial x_{i}}=0
$$

Navier-Stokes

$$
\frac{\partial \bar{\rho} \widetilde{u}_{i}}{\partial t}+\frac{\partial \bar{\rho} \widetilde{u}_{i} \widetilde{u}_{j}}{\partial x_{j}}=-\frac{\partial \bar{p}}{\partial x_{i}}+\frac{\partial \bar{\tau}_{i j}}{\partial x_{j}}-\frac{\partial \tau_{i j}^{*}}{\partial x_{j}},
$$

Species Mass Fractions

$$
\frac{\partial \bar{\rho} \widetilde{Y}_{\alpha}}{\partial t}+\frac{\partial \bar{\rho} \widetilde{u}_{j} \widetilde{Y}_{\alpha}}{\partial x_{j}}=\frac{\partial}{\partial x_{j}}\left(\frac{\mu}{\sigma} \frac{\partial \widetilde{Y}_{\alpha}}{\partial x_{j}}\right)+\frac{\partial J_{j}^{\alpha}}{\partial x_{j}}+\overline{\rho \dot{\omega}_{\alpha}},
$$

Enthalpy

$$
\frac{\partial \bar{\rho} \widetilde{h}}{\partial t}+\frac{\partial \bar{\rho} \widetilde{u}_{j} \widetilde{h}}{\partial x_{j}}=\frac{\partial}{\partial x_{j}}\left(\frac{\mu}{\sigma} \frac{\partial \widetilde{h}}{\partial x_{j}}\right)-\frac{\partial J_{j}}{\partial x_{j}}+\dot{q}
$$

where $\tau_{i j}^{*}=\bar{\rho}\left(\widetilde{u_{i} u_{j}}-\widetilde{u}_{i} \widetilde{u}_{j}\right)$ is the sub-filter scale stress tensor which is here determined by means of the dynamic Smagorinsky model

$$
\tau_{i j}^{*, a}=2 \mu_{s g s} \widetilde{S}_{i j}
$$

The quantities $\mu_{s g s}=\bar{\rho}\left(C_{s} \Delta\right)^{2}\left\|\widetilde{S}_{i j}\right\|$, and $\widetilde{S}_{i j}$ denote the sub-grid scale viscosity and the resolved rate of strain tensor $\widetilde{S}_{i j}$, respectively. $\|\cdot\|$ is the Frobenius norm. The filter scale $\Delta$ is taken to be the cube root of the local grid cell volume ${ }^{1}$ which varies with position. Hence, spatial filtering and differentiation will generally not commute although for smoothly varying mesh spacings any resulting error is likely negligible. The parameter $C_{s}$ is calculated using the dynamic procedure of Piomelli and Liu [27]. The isotropic part of the viscous and sub-filter stresses is absorbed into the pressure. The filtered equations for the mass fractions of the $N$ chemical species and the enthalpy of the mixture contain unknown sub-grid scale fluxes and the filtered net species formation rates. These are modelled by means of the PDF approach.

\footnotetext{
1 When the filter width is linked to the mesh spacing the unresolved motions are referred to as sub-gridscale motions
} 


\subsection{Sub-filter PDF model}

Following Gao and O'Brien [13], an equation describing the evolution of the density-weighted sub-filter PDF for the species mass fraction and the enthalpy of the mixture can be derived. This equation contains unknown terms which represent the sub-filter scale transport of the PDF and sub-filter scale mixing. In the present work these are approximated by a Smagorinsky type gradient model and the Linear Mean Square Estimation (LMSE) closure, respectively. The modelling of the sub-filter scale mixing is difficult and no entirely satisfactory model exists. The LMSE mixing model used in the present work constitutes a reasonable choice between simplicity and predictive capabilities. With these models the sub-filter PDF transport equations for the species mass fractions and enthalpy of the mixture have the following generic form

$$
\left.\begin{array}{rl} 
& \bar{\rho} \frac{\partial \widetilde{P}_{s g s}(\underline{\psi})}{\partial t}+\bar{\rho} \widetilde{u}_{j} \frac{\partial \widetilde{P}_{s g s}(\underline{\psi})}{\partial x_{j}}+\sum_{\alpha=1}^{N} \frac{\partial}{\partial \psi_{\alpha}}\left[\bar{\rho} \dot{\omega}_{\alpha}(\underline{\psi}) \widetilde{P}_{s g s}(\underline{\psi})\right] \\
= & \frac{\partial}{\partial x_{j}}\left[\left(\frac{\mu}{\sigma}+\frac{\mu_{s g s}}{\sigma_{s g s}}\right) \frac{\partial \widetilde{P}_{s g s}(\underline{\psi})}{\partial x_{j}}\right] \\
- & \frac{C_{d}}{\tau_{s g s}} \sum_{\alpha=1}^{N} \frac{\partial}{\partial \psi_{\alpha}}\left[\bar{\rho}\left(\psi_{\alpha}-\widetilde{\phi}_{\alpha}(\mathbf{x}, t)\right) \widetilde{P}_{s g s}(\underline{\psi})\right]
\end{array}\right\}
$$

in which $\widetilde{\phi}_{\alpha}$ denotes either the specific mole number of the $\alpha$-th chemical species or the enthalpy. The quantity $\underline{\psi}$ designates the phase space of the scalar $\widetilde{\phi}_{\alpha}$. In the present work the constants $\sigma_{s g s}$ and $\bar{C}_{d}$ are set to 0.7 and 2.0, respectively. The sub-filter mixing time scale $\tau_{s g s}$ is given by

$$
\tau_{s g s}=\frac{\bar{\rho} \Delta^{2}}{\mu+\mu_{s g s}} .
$$

In the derivation of (6) we have assumed that the Schmidt numbers $\sigma$ are constant, equal for all chemical species and equal to the Prandtl number. This corresponds to a Lewis number of unity.

\subsection{Eulerian stochastic field method}

Eq. (6) is solved using the Eulerian stochastic field method based on an Itō interpretation of the stochastic integral. The PDF $\widetilde{P}_{s g s}(\psi)$ is represented by an ensemble of $N_{s}$ stochastic fields for each of the species mass fractions and the enthalpy, namely $\xi_{\alpha}^{n}(\mathbf{x}, t)$, with $1<$ $n<N_{s}$. The evolution of the Eulerian stochastic fields is described by

$$
\left.\begin{array}{rl}
\bar{\rho} d \xi_{\alpha}^{n}= & -\bar{\rho} \widetilde{u}_{i} \frac{\partial \xi_{\alpha}^{n}}{\partial x_{i}} d t+\frac{\partial}{\partial x_{i}}\left[\Gamma \frac{\partial \xi_{\alpha}^{n}}{\partial x_{i}}\right] d t+\bar{\rho} \sqrt{\frac{2 \Gamma}{\bar{\rho}}} \frac{\partial \xi_{\alpha}^{n}}{\partial x_{i}} d W_{i}^{n} \\
& -\frac{C_{d} \bar{\rho}}{2 \tau_{s g s}}\left(\xi_{\alpha}^{n}-\widetilde{\phi}_{\alpha}\right) d t+\bar{\rho} \dot{\omega}_{\alpha}^{n} d t,
\end{array}\right\}
$$

where $\Gamma$ and $d W_{i}$ represent the total diffusion coefficient and the increments of a vector Wiener process which is different for each stochastic field but independent of the spatial position. This stochastic process has no influence on the first moments (ie filtered mean values). The Wiener process is approximated by time-step increments $\eta_{i}^{n} \sqrt{d t}$, where $\eta_{i}^{n}$ is 
a $\{-1,+1\}$ dichotomic vector. The solutions of Eq. (8) preserve any bounded properties of the scalar in question as the gradient of the scalar will tend to zero when the value of the scalar approaches extrema, and therefore the stochastic contribution will tend to zero. The reader should consult [20] for a thorough discussion of the Eulerian stochastic field method.

\section{Experiment}

The experimental configuration investigated here has been developed by Balachandran [5] and is aimed at analysing the response of acoustically excited flames. A simple diagram of the experimental apparatus is displayed in Fig. 1. The working fluid is a mixture of ethylene $\left(\mathrm{C}_{2} \mathrm{H}_{4}\right)$ and air which is premixed to an equivalence ratio of 0.55 and supplied through an injection unit in the bottom section of the apparatus. Diametrically opposed loudspeakers downstream of the injection unit are used to excite the mass flux harmonically with a forcing amplitude, $A$, and a forcing frequency, $f$. The bulk-averaged axial velocity of the mixture at the inlet of the combustion chamber, $v_{b}$, is $10 \mathrm{~m} / \mathrm{s}$. Together with the base diameter of the conical bluff body, $D_{b}=25 \mathrm{~mm}$, this yields a cold flow Reynolds number of approximately 19,000 . The bulk averaged velocity $v_{b}$ and diameter $D_{b}$ are used subsequently as reference values for non-dimensionalising the results arising from the simulations. The combustion chamber is open to the atmosphere at the downstream end. Although the response of the flame has been previously investigated for a range of excitation parameters, the present simulations focus on the case corresponding to $A=0.60 v_{b}$ and $f=0.4 v_{b} / D_{b}$.

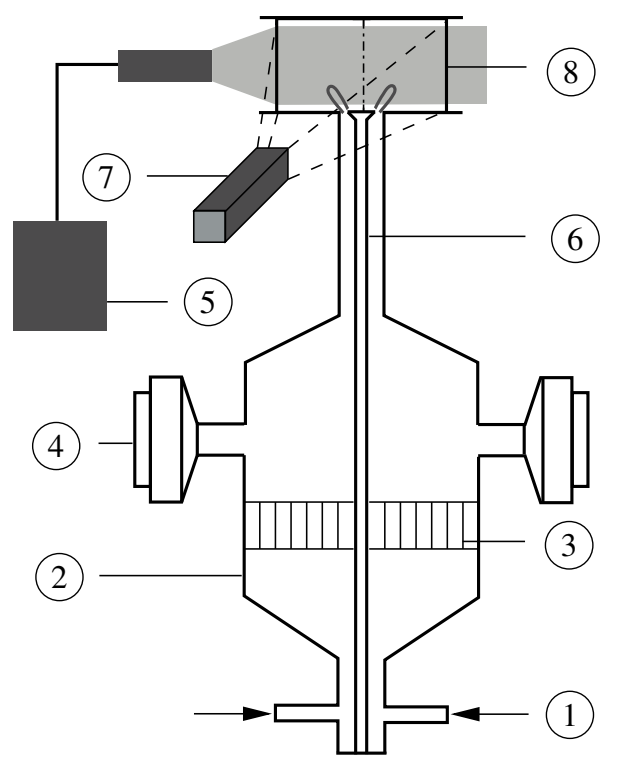

Fig. 1 Diagram of Balachandran's apparatus for planar laser-induced fluorescence imaging in an oscillating flame (not to scale); 1: ethylene-air injection, 2: plenum chamber, 3: fow straightener, 4: loudspeaker, 5: laser, 6: bluff body with holder, 7: camera, 8: quartz tube.

Various measurement techniques have been employed to determine the variation of the heat release rate. Amongst them are chemiluminescence measurements of the chemically 
excited hydroxyl radical $\left(\mathrm{OH}^{*}\right)$, and planar laser-induced fluorescence of the hydroxyl radical $(\mathrm{OH})$ and formaldehyde $\left(\mathrm{CH}_{2} \mathrm{O}\right)$. The laser sheets for the $\mathrm{OH}$ and $\mathrm{CH}_{2} \mathrm{O}$ fluorescence imaging coincide with the median plane of the combustion chamber (indicated by the grey shaded area in Fig. 1). Within Heat Release Rate (HRR) imaging the $\mathrm{OH}$ and $\mathrm{CH}_{2} \mathrm{O}$ fluorescence signals are recorded simultaneously and multiplied with each other to yield an estimate for the production rate of the formyl radical (HCO). The formation of $\mathrm{HCO}$ involves reactions which are considered to dominate the overall heat release rate and also plays an important role in the final stages of the production of carbon monoxide (CO). The Flame Surface Density (FSD) is an additional measure of the heat release rate and is computed from the measurements from the raw images of the $\mathrm{OH}$ fluorescence. Chemiluminescence of $\mathrm{OH}^{*}$ is frequently used as an integral measure for the total heat release rate since the emission intensity, $I$, is found to scale linearly with the fuel consumption rate. Both HRR and FSD serve to provide a spatial resolution of the regions with high chemical activity. It is important to note that the intensity scale of the fluorescence and chemiluminescence signals is arbitrary. The measurements are thus scaled by means of the time and spatial average of the intensity. Experimental data of $\mathrm{OH}$ chemiluminescence and FSD are provided by Balachandran [5], whereas Ayoola [4] provide HRR imaging data.

In addition to the experiment on oscillating flames, Ahmed at al. [1] and Ayache et al. [3] have conducted measurements to quantify the velocity field of the cold flow in this same experimental apparatus. The former has provides measurements of the velocity field in the absence of acoustic excitation and the latter provides the axial velocity of the acoustically excited flow for the above mentioned excitation parameters. The working fluid was air under atmospheric conditions. This provides a convenient way to sequentially validate the LES method. To characterise the periodic evolution of the heat release rate and allow for comparison with the measurements the simulations have been phase averaged with respect to the excitation signal. The notation $\langle\cdot\rangle_{t},\langle\cdot\rangle_{x}$ and $\langle\cdot\rangle_{\gamma}$ is introduced to distinguish between time, spatial and phase averages respectively. $\gamma \in[0,1]$ denotes the normalised phase and is computed from the simulation by means of $\gamma=\bmod (f \times t, 1)$.

\section{Simulation details}

\subsection{Computational grid}

Fig. 2 presents the geometry of the computational domain and identifies the locations of the boundary conditions. 


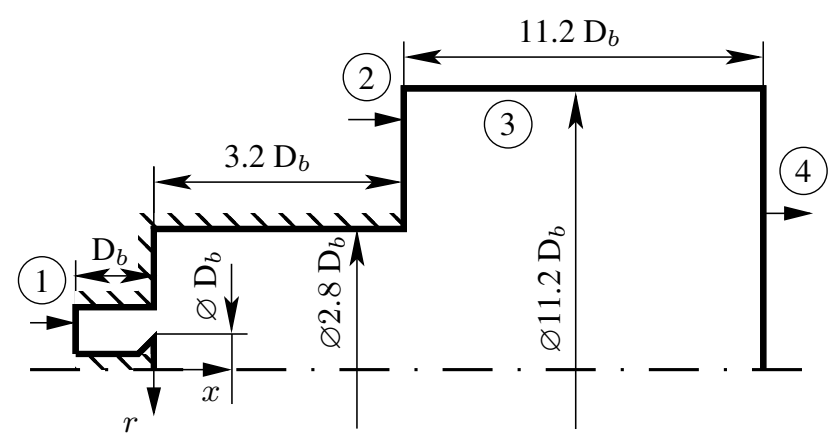

\ (5)

Fig. 2 Sketch of the computational domain and boundary conditions for the simulation (not to scale). The boundary conditions are as follows: 1: ethylene-air inlet, 2: air co-fow, 3: free slip boundary, 4: zero-gradient outlet, 5: no-slip walls.

Sections of the computational grid are shown in Fig. 3. The recommendations of Ayache et al. [3] have been followed and the computational domain is discretised with approximately two million cells. The grid is stretched along the axial and radial directions to allow for a higher concentration of grid nodes around the bluff body and the inlet of the combustion chamber. The mesh stretching factor is everywhere maintained below $5 \%$. The minimum axial and radial grid spacing are located in proximity to the inlet of the combustion chamber and have a value of $0.004 D_{b}$ and $0.007 D_{b}$, respectively.

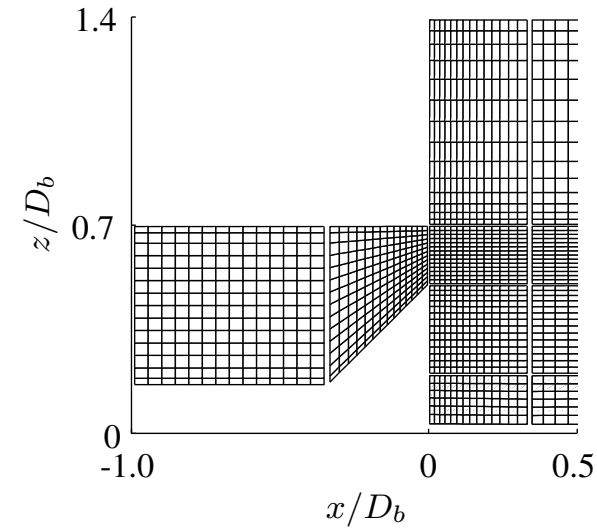

(a)

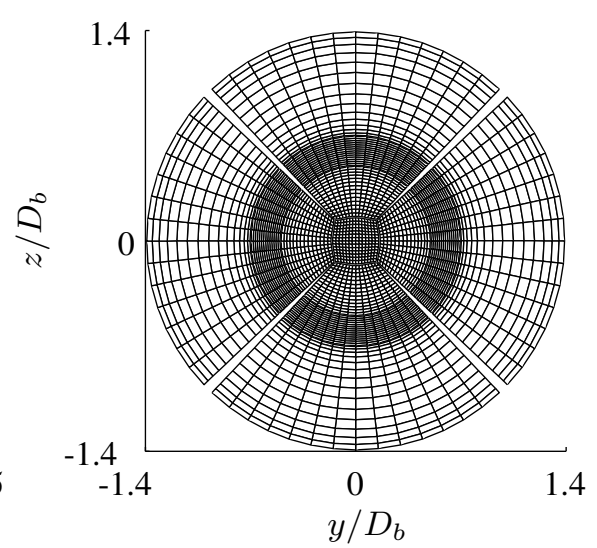

(b)

Fig. 3 Computational grid used in the simulations; only every second grid line is shown for clarity.

\subsection{Boundary conditions}

LES requires turbulent velocity inlet conditions which are here generated with the method of Klein et al. [23,9]. In order to obtain realistic inflow conditions the velocity field upstream of the bluff body is characterised by means of the time-averaged velocity components and the 
Reynolds stress tensor. These quantities have been determined in a separate auxiliary simulation using the Reynolds-Averaged Navier-Stokes (RANS) solver and the Reynolds Stress Model (RSM) available in the commercial software package STAR-CCM+. The computational domain of the auxiliary simulation comprises the two concentric ducts upstream of the bluff body and has a length of $10 D_{b}$. The turbulent flow in the annular gap between the two ducts is presumed to be fully developed and therefore periodic conditions are applied at the stream wise boundaries. The length of the computational domain was chosen to ensure that the two-point correlations of the velocity field attain zero at the midpoint between the two stream wise boundaries. A forcing term is applied everywhere in the domain to maintain the given mass flow rate consistent the experiment. The method of Klein et al. also requires case-specific length scales for each coordinate direction in the inlet plane, $L_{y}$ and $L_{z}$, and an associated time scale, $t_{s}$, with the following values $L_{y}=L_{z}=0.15 D_{b}$ and $t_{s}=0.0008 D_{b} / v_{b}$ being selected. $L_{y}$ and $L_{z}$ are estimates of the integral length scale and $t_{s}$ is assumed to be ten times the estimated integral time scale. Reference parameters have been taken from an experimental study of R. Zhou on the flow field of a non-reacting mixture in the Cambridge stratified swirl burner. Fig. 4 presents the spatial distribution of the instantaneous turbulent kinetic energy in the inlet section and the computed power spectral density of the kinetic energy at $x=0$ and $r=0.6 D_{b}$.

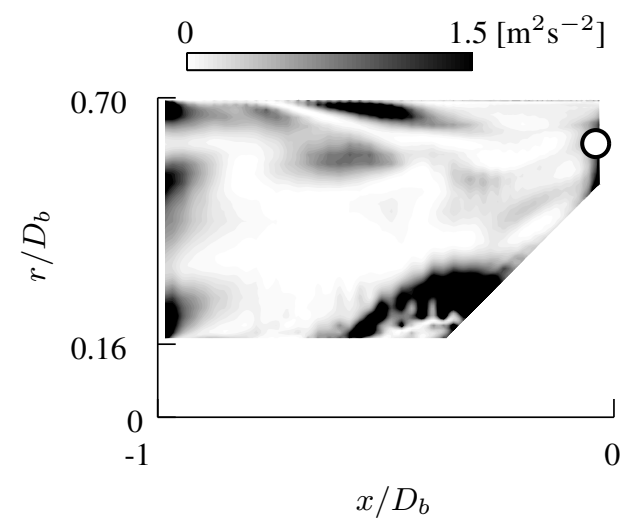

(a)

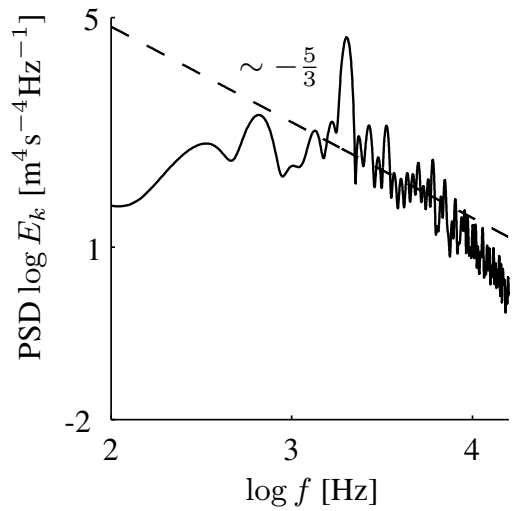

(b)

Fig. 4 Predicted flow field in the inlet section; (a) instantaneous turbulent kinetic energy in the median plane, (b) power spectral density of the kinetic energy at $x=0, r=0.6 D_{b}$. The power spectral density has been obtained by subdividing the time signal into eight windows with $50 \%$ overlap. Each segment is windowed with the Hamming window function. The white point in (a) marks the monitor position for the kinetic energy.

Turbulent structures, resulting from the prescribed inflow conditions, readily develop in the inlet duct and this is also confirmed by the observed inertial subrange in the energy spectrum, Fig. 4. In the case of the oscillating flows a sinusoid with the excitation parameters $A=0.60 v_{b}$ and $f=0.4 v_{b} / D_{b}$ is superimposed on the turbulent velocity signal, consistent with the experiment. The fuel and oxidiser streams are premixed far upstream of the combustion chamber $\left(\sim 80 D_{b}\right)$ and therefore the mass fractions of the unburnt mixture are assumed homogeneous across the inlet plane. The temperature at the inlet is $293 \mathrm{~K}$.

In addition, it is assumed that the walls of the combustion chamber are adiabatic since the heating flux through the walls is generally small compared to the heat release rate. In 
the initial runs with the computational domain extending only to the downstream end of the combustion chamber artificial reflections in the velocity field were encountered and it was therefore decided to incorporate part of the surroundings into the computational domain. The fluid being entrained by the jet at the outlet of the combustion chamber is represented by an air co-flow at a temperature of $293 \mathrm{~K}$. This co-flowing stream was not present in the experiment but was included to avoid large unphysical recirculation zones in the outer region, as is common practice in the simulation of jet flows [11]. The axial co-flow velocity was chosen such that the time-averaged mass flux is $10 \%$ of the mass flux at the outlet of the combustion chamber. Decreasing the mass flux of the co-flow to $5 \%$ of the mass flux at the outlet of the combustion chamber did not alter the flow response in the combustion chamber.

\subsection{Solver and averaging procedure}

The in-house flow solver BOFFIN-LES is employed for all the computations. It comprises a block-structured, boundary-conforming finite volume code with second-order discretisation schemes in space and time. The code utilises a co-located storage arrangement and is fully parallelised by means of domain decomposition and MPI message passing routines. The convective terms of the momentum balance are discretised with an energy-conserving scheme and all other spatial derivatives except for the convection terms in the scalar balance equations are discretised with second-order central differences. To avoid unphysical overshoots, a Total Variation Diminishing (TVD) scheme is used to discretise the convective terms in the scalar equations. The momentum and continuity equations are solved using a pressure correction method. A fractional step method is used for the stochastic field equations with a Newton-method being used to integrate the stiff chemical rate equations. The integration of the chemical reactions is additionally parallelised to speed up the computation. The chemical reaction of ethylene with air is represented by means of the detailed but reduced reaction mechanism of Luo et al. [24]. This mechanism involves 22 molecular species and 18 global reactions and has been obtained by a systematic reduction of a detailed mechanism comprising 75 species and 529 reactions.

Table 1 Performance characteristics

\begin{tabular}{lccc}
\hline Simulation & $\begin{array}{c}\text { Non-oscillating } \\
\text { cold flow }\end{array}$ & $\begin{array}{c}\text { Oscillating cold } \\
\text { flow }\end{array}$ & Oscillating flame \\
\hline \# MPI processes & 81 & 81 & 81 \\
\# Stochastic fields & - & - & 4 \\
\# Time steps & 500 & 500 & 500 \\
Time step size [s] & $5 \cdot 10^{-6}$ & $5 \cdot 10^{-6}$ & $5 \cdot 10^{-6}$ \\
\hline Running time [s] & $7.3 \cdot 10^{3}$ & $6.3 \cdot 10^{3}$ & $7.6 \cdot 10^{4}$ \\
\hline
\end{tabular}

Each simulation is carried out on eleven computing nodes with Infiniband interconnects. Each node comprises two 2.5 Ghz Quad core Intel Xeon processors. Table 1 summarises the performance characteristics of each computation for a sample simulation time of 500 time steps. The running time associated with the simulation of the oscillating flame is an order of magnitude longer than those associated with the simulation of the cold flows. This significant increase is explained by the costly integration of the stiff chemical rate equations which 
consume approximately $70 \%$ of the total running time despite the parallelisation of the integration work. The additional solution of $4(N+1)$ differential equations in the simulation of the oscillating flame also contributes to an increased running time.

To reach a fully developed flow before computing the statistics each simulation was run for sixty characteristic time periods, $t_{b}=D_{b} / v_{b}$. In the simulation of the non-oscillating and oscillating cold flows, statistics were gathered over a period of $450 t_{b}$. Such a long integration time was needed to obtain sufficiently smooth results for the second moments, especially in the recirculation zones where the velocities are low. Due to the increased computational demand of the oscillating flame collected phase-averaged statistics for this case were collected over a reduced period of $125 t_{b}$, which corresponds to 50 cycles associated with the applied forcing frequency. This integration time is somewhat shorter than the integration time used in the simulation of the cold flows but yields sufficiently smooth statistics, see Fig. 5. It must also be noted that the phase-averaged statistics settle faster in the oscillating flame due to the decreased turbulence intensity. Spatial averaging in the circumferential direction is additionally performed to reduce noise in the statistics.

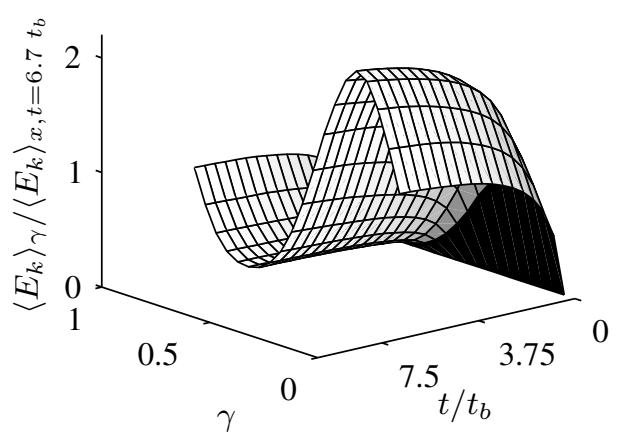

(a)

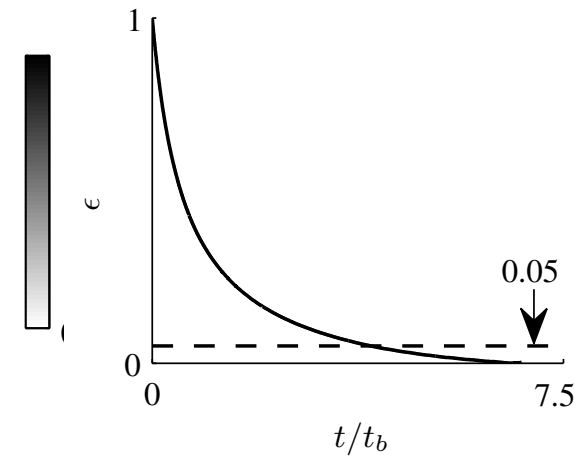

(b)

Fig. 5 Convergence of the phase-averaged statistics for the kinetic energy at $x=0, r=0.6 D_{b}$; (a) nondimensional kinetic energy as a function of $\gamma$ and $t / t_{b}$ coloured with the deviation from the final profile at $t=6.7 t_{b}$, (b) deviation from the final value at $t=6.7 t_{b}$ as a function of $t / t_{b}$ for $\gamma=0.25$.

\section{Results and discussion}

\subsection{Non-oscillating cold flow}

Fig. 6 shows contour plots of the instantaneous and time-averaged axial and radial velocities. For later reference, Fig. 6 (a) also shows five axial stations and six probe locations at which experimental data of the time-averaged and phase-averaged flow field are available. The axial stations and probe locations are listed in Table 2 and Table 3, respectively.

Table 2 List of the axial stations in the experiment of Ahmed et. al [1].

\begin{tabular}{cccccc}
\hline Name & $X_{1}$ & $X_{2}$ & $X_{3}$ & $X_{4}$ & $X_{5}$ \\
\hline$x / \mathrm{D}_{b}$ & 0.22 & 0.62 & 1.00 & 1.40 & 2.00 \\
\hline
\end{tabular}


A large zone of recirculating fluid is established downstream of the conical bluff body and is referred to as the Central Recirculation Zone (CRZ). The dimensions of the CRZ in the axial and radial directions are $1.1 D_{b}$ and $0.9 D_{b}$, respectively. The time-averaged mean axial velocity has a minimum in the CRZ with a value of $-0.5 v_{b}$ and a maximum in the annular jet with a value of about $+1.2 v_{b}$. A similar flow pattern is evident in the contour plot of the instantaneous axial velocity. The dimensions of the CRZ agree with those observed experimentally by Balachandran [5] using smoke visualisation. Shear layers develop on the inner and outer radius of the annular jet, indicated by sharp gradients of the mean axial and radial velocity near the combustor inlet.
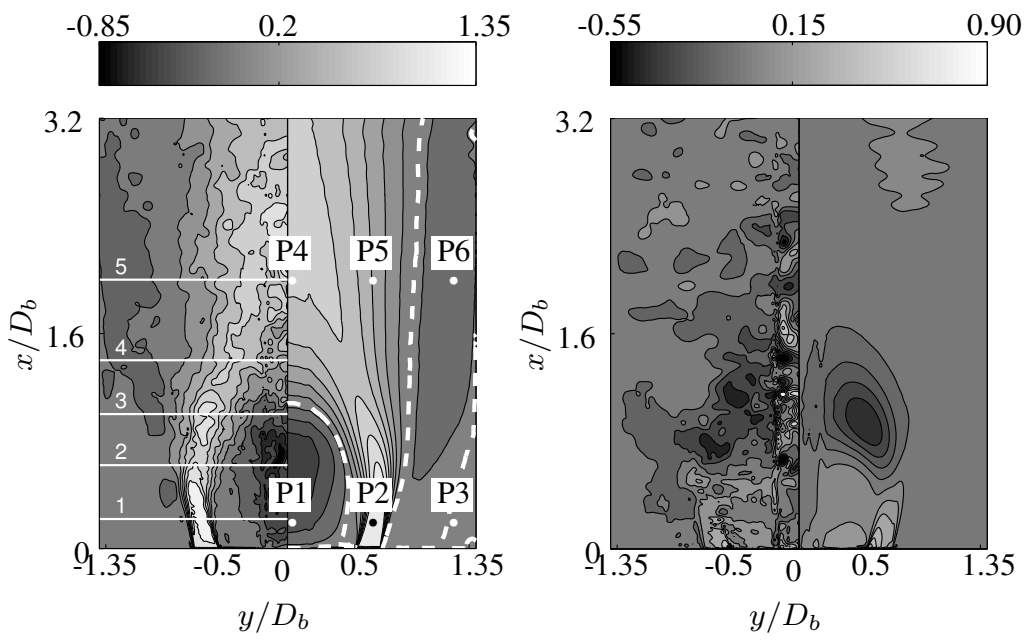

Fig. 6 Contour plots of the computed velocity field, left half: instantaneous field, right half: time-averaged mean field; (a) axial velocity $v_{x} / v_{b}$, (b) radial velocity $v_{r} / v_{b}$. The white dashed line represents the zero contour.

The mean radial velocity has its minimum in the expanding annular jet at the rear of the recirculation zone with $-0.4 v_{b}$ and a maximum, with a value of $0.5 v_{b}$, in the annular jet at the inlet of the combustion chamber. Strong fluctuations of the radial velocity occur near the centerline of the combustion chamber, but do not lead to an increased time-averaged mean value. In fact, the instantaneous radial velocity seems to alternate along the axial direction, suggesting that these fluctuations will vanish in the time average. The outer shear layer does not re-attach at the wall of the combustion chamber. Instead, a large annular recirculation zone which extends to the exit of the combustion chamber, referred to as Outer Recirculation Zone (ORZ), occupies the region between the wall and the outer shear layer. This exerts a significant influence on the choice of the size of the computational domain, since it prohibits the imposition of appropriate boundary conditions at the exit of the combustion chamber.

Table 3 List of the probe positions in the experiment of Ayache et. al [3].

\begin{tabular}{lcccccc}
\hline Name & $\mathrm{P} 1$ & $\mathrm{P} 2$ & $\mathrm{P} 3$ & $\mathrm{P} 4$ & $\mathrm{P} 5$ & $\mathrm{P} 6$ \\
\hline$x / \mathrm{D}_{b}$ & 0.2 & 0.2 & 0.2 & 2.0 & 2.0 & 2.0 \\
$r / \mathrm{D}_{b}$ & 0.0 & 0.6 & 1.2 & 0.0 & 0.6 & 1.2 \\
\hline
\end{tabular}


Fig. 7 shows the radial profiles of the simulated time-averaged mean and root-meansquare of the axial velocity together with corresponding experimental data at the stream wise stations listed in Table 2. The simulated values compare well with the experimental data at all axial stations. The dimensions of the recirculation zone are in excellent agreement with the measurements. Both the spreading of the annular jet and the decaying turbulence levels in the shear layers are well captured.

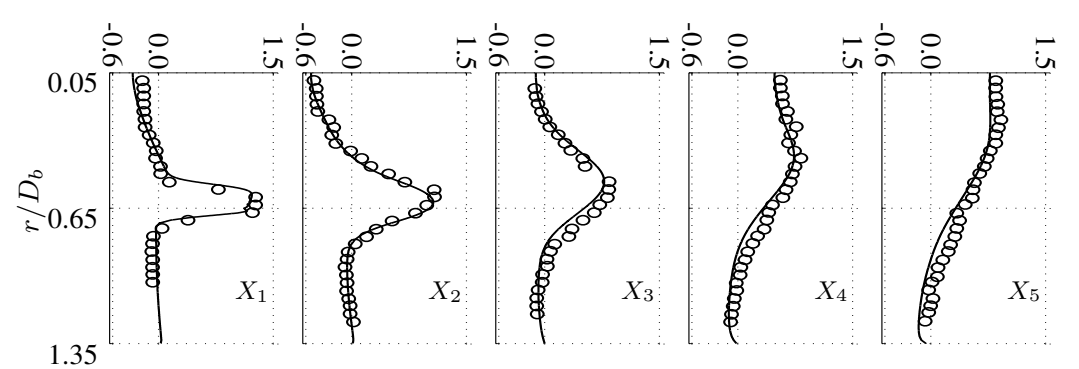

(a) $\left\langle v_{x}\right\rangle_{t} / v_{b}$

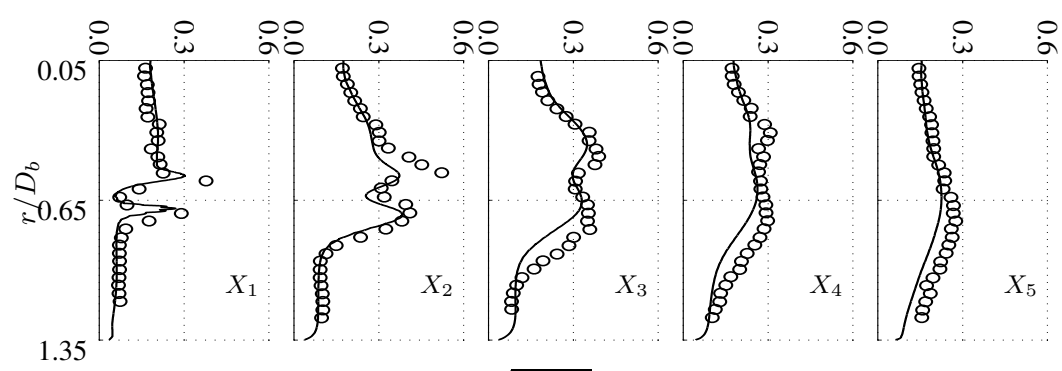

(b) $\sqrt{\left\langle v_{x}^{\prime \prime 2}\right\rangle_{t}} / v_{b}$

Fig. 7 Statistical profiles for the axial velocity, symbols: experiment [1], solid lines: simulation; (a) timeaveraged mean, (b) root-mean-square. 


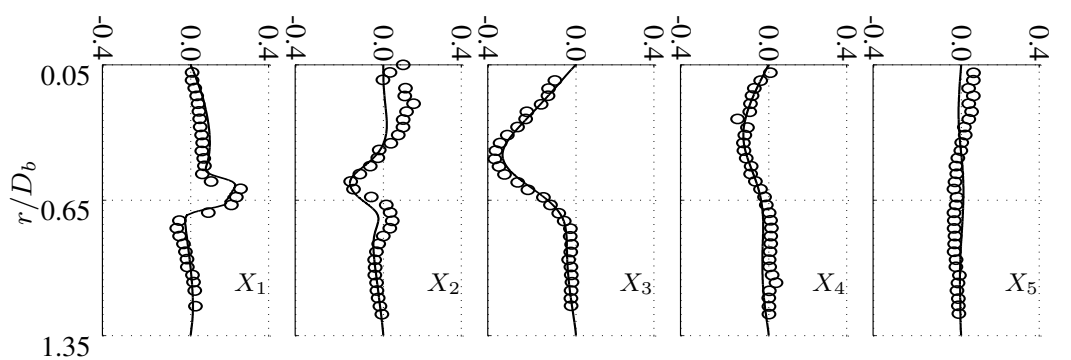

(a) $\left\langle v_{r}\right\rangle_{t} / v_{b}$

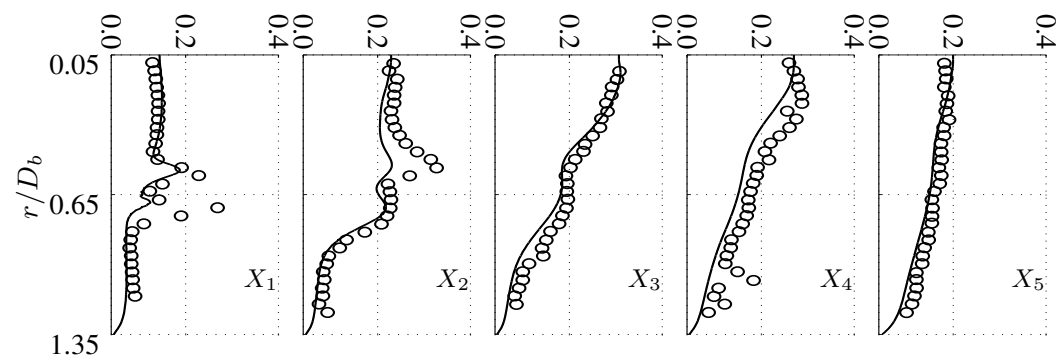

(b) $\sqrt{\left\langle v_{r}^{\prime \prime 2}\right\rangle_{t}} / v_{b}$

Fig. 8 Statistical profiles for the radial velocity, symbols: experiment [1], solid lines: simulation; (a) timeaveraged mean, (b) root-mean-square.

Radial profiles of the time-averaged mean and root-mean-square of the radial velocity at the same axial stations are shown in Fig. 8. The simulated results generally compare well with experimental data. The shape of the widening annular jet is well captured with respect to the measurements. However, the simulated turbulence levels in the outer shear layer near the combustor inlet are too low by approximately $70 \%$. This discrepancy in proximity to the inlet might be attributed to the intermittent nature of the flow in this region which could potentially lead to an excess of sub-grid diffusion caused by increased values of the computed eddy viscosity. In spite of this the levels of the fluctuations recover further downstream. Despite the relatively short length of the annular duct upstream of the combustion chamber, the imposed turbulent inflow velocity yields reasonable agreement with the experimental data near the combustor inlet.

\subsection{Oscillating cold flow}

The upper halves of Fig. 9 (a)-(c) show the evolution of the simulated streamline pattern based on the phase-averaged velocity field. The observed flow pattern is reminiscent of the non-oscillating cold flow, for example a zone of recirculating fluid forms downstream of the conical bluff body and in proximity to the cylindrical wall of the combustion chamber. In this case, however, there persist two distinct outer recirculation zones at the beginning of each cycle which eventually merge into one larger one towards the end of the cycle. A widening annular jet is formed between the CRZ and the upstream ORZ, generating shear layers on both sides of the annular jet. 


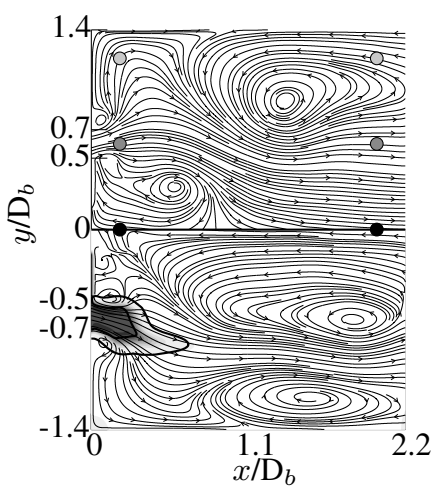

(a) $\gamma=0.0$

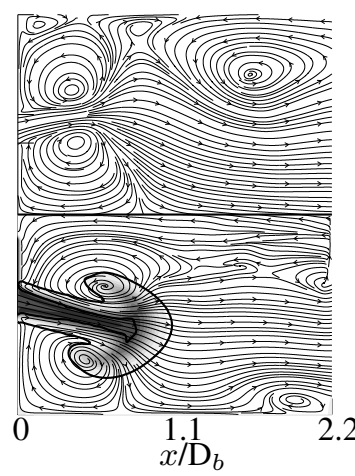

(b) $\gamma=0.33$

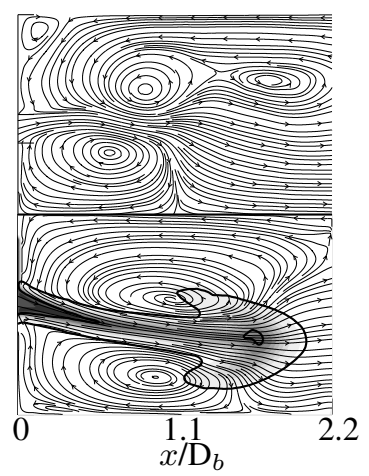

(c) $\gamma=0.66$

Fig. 9 Streamline pattern for the oscillating inert air flow (upper half) and the reacting ethylene-air mixture (lower half). The thick black solid line in the lower half represents the flame contour. The temperature field is coloured with grayscale. Dark and bright levels represent low and high temperatures, respectively. The six grayscale points in (a) mark the probe positions listed in Table 3

Fig. 10 shows the experimentally determined and the simulated phase-averaged axial velocity component at the six probe positions. In general, the computed results compare well with the measurements at all positions. It is worth noting that good agreement is obtained at $\mathrm{P} 2$ in the range of $0.25 \leq \gamma \leq 0.7$, which is the position closest to the inlet of the computational domain and an indicator for the adequacy of the imposed velocity field. The slight underestimate of the time average is approximately the same as in the non-oscillating cold flow, see the profile at $X_{1}$ in Fig. 7 (a). At P5 and P6, the simulated axial velocity is somewhat too low but the overall features are reproduced satisfactorily. From both Fig. 9 and Fig. 10 it becomes clear that the CRZ and ORZ are excited at the frequency of the oscillating annular jet, albeit at a much smaller amplitude. The phase-averaged axial extent of the central recirculation zone, for example, increases only slightly from $0.7 D_{b}$ to $1.1 D_{b}$ in the course of the cycle. At the same time, the simulated axial velocity at P1 shows a small variation about the time average. There is also no observable phase shift between the excitation signal at the inlet and the response of the axial velocity at the downstream positions $\mathrm{P} 4$ to $\mathrm{P} 6$, which implies that the time scale associated with the convection of the imposed velocity oscillation is approximately the same as the time period associated with the forcing frequency. 

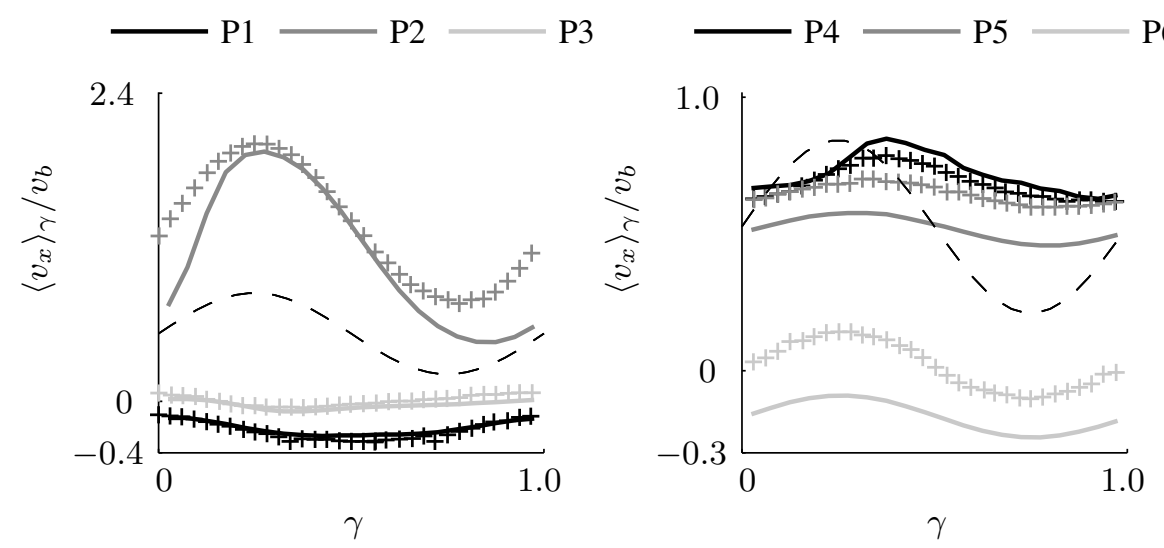

Fig. 10 Profiles of the phase-averaged axial velocity; symbols: experimental data of Ayache et al. (2010), solid lines: simulation, dashed line: excitation signal.

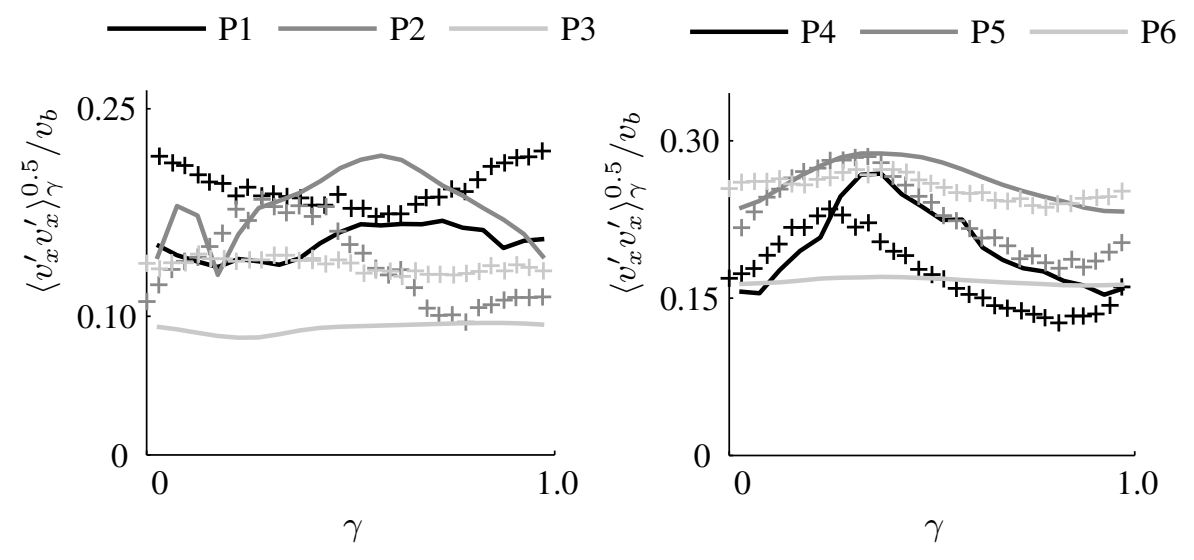

Fig. 11 Profiles of the phase-averaged fluctuations of the axial velocity; symbols: experimental data of Ayache et al. (2010), solid lines: simulation, dashed line: excitation signal.

Fig. 11 presents a comparison between the experimentally determined and the simulated phase-averaged fluctuations of the axial velocity. The agreement is less satisfactory than in the previous case. Noticeable discrepancies between the computed and experimentally determined profiles are found at the upstream positions P1 to P3. The streamline pattern (Fig. 9) reveals that the shear layers between the radially widened annular jet and the recirculation zones are passing periodically through the probe positions P2 and P3. One possible explanation for the discrepancies is that the dynamic Smagorinsky model is not able to reproduce the intermittent nature of the flow in this region. Indeed, da Silva [10] has demonstrated that the dynamic Smagorinsky model has deficiencies in the zone separating the turbulent and non-turbulent intermittency region in a jet flow. According to his findings, the standard Smagorinsky model should perform slightly better in this region. The simulated and measured profiles are overall better agreement at the downstream positions P4 to P6. 
5.3 Oscillating flame

The evolution of the reaction zone together with the streamline pattern of the flow is presented in Fig. 9 (a)-(c). The contour level for the visualisation of the reaction zone is $15 \%$ of the maximum heat release rate. It has to be acknowledged that the variation of the contour level affects slightly the observed shape of the reaction zone at the downstream end of the flame. This can be attributed to the gradient of the heat release rate being lower at the downstream end of the flame than in the shear layers between the annular jet and the recirculation zones. The criterion suffices, however, to explain some of the main features of the flow. The flow pattern of the reacting mixture exhibits both the CRZ and ORZ, albeit with different axial extents and shifted with respect to the phase when compared to the cold flow results. It is observed that, in the case of the reacting mixture, the axial extents of the CRZ and ORZ vary more significantly during the cycle. The flame elongates by a factor of four in the axial direction and becomes thinner in the radial direction in the course of the cycle. The CRZ and ORZ deform the flame in the radial direction resulting in the distinct mushroom-like structure at $\gamma=0.33$. It is worth noting that the temperature distribution in the reaction zone along the middle axis of the annular jet reaches a local maximum at $x / D_{b}=1.2$ and $y / D_{b}=-0.7$ in the later stages of the cycle $(\gamma=0.66)$.

The shape of the reaction zone is compared with experimental data in Fig. 12. Despite scatter in the experimental data the comparison serves to identify potential deviations between experiment and simulation. Note that the reference points for the phase-averaging procedure differ between experiment and simulation. In the experiment the beginning of each cycle for the phase average was determined with reference to the forcing signal used to excite the loudspeakers in the plenum chamber while the phase average in the simulation was carried out with respect to the imposed sinusoidal fluctuation at the inflow boundary. The experimental data is phase-shifted with respect to the predicted results by matching the flame shape at the minimum spatial extent of the reaction zone at the beginning of the cycle. For $0 \leq \gamma<0.5$ the results of the simulation are in good agreement with experimental data. The closest agreement between experiment and simulation is achieved at the phase $\gamma=0.33$, when the flame is freely propagating and not affected by the presence of the combustor wall. The angle of the flame with respect to the centre axis is well reproduced in the simulation and the predicted characteristic mushroom-like structure at the downstream end of the flame is also clearly observed in the FSD data. A rather significant deviation becomes obvious at $\gamma=0.66$. The flame appears deflected towards the centre axis in the simulation, whereas HRR and FSD both suggest that the flame is in contact with the wall. The flame deflection is likely to be related to the applied boundary conditions since the walls do not admit heat transfer. This assumption is not valid when the flame approaches the wall. As a result of the incorrect boundary treatment the stagnation point at the combustor wall and also the annular-shaped ORZ are likely shifted further downstream, whereby the latter directs the flame away from the wall. Indeed, the observation that the tail of the reaction zone remains approximately parallel to the wall in the later stages of the cycle $(\gamma \geq 0.8)$ implies that the ORZ functions as a hydrodynamic "cushion" between the flame and the wall. Another phenomenon for which there is no evidence in the experimental data is the local extinction of the flame at $\gamma=0.85$. We have verified that this is not an artifact of the flame visualisation by reconstructing the contour of the reaction zone at different levels of the heat release rate. The obtained results suggest that the tail of the flame extinguishes in the annular jet at approximately $x / D_{b}=1.9$ and $r / D_{b}=0.9$, which divides the flame into two parts. 


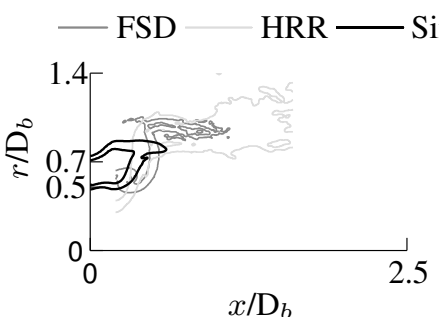

(a) $\gamma=0.0$

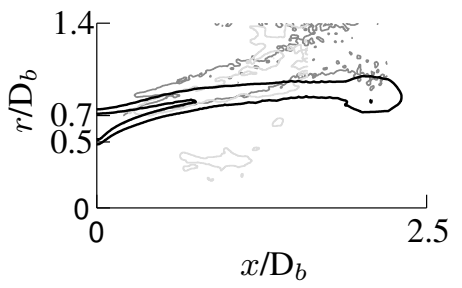

(d) $\gamma=0.80$

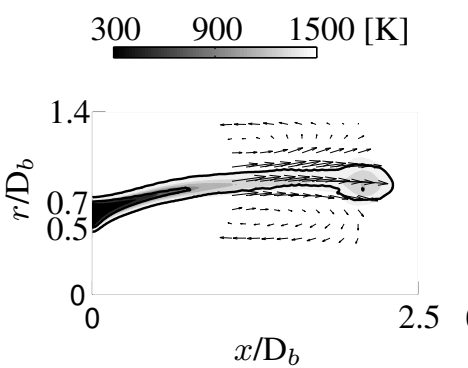

(g) $\gamma=0.80$

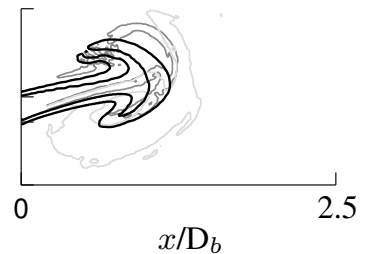

(b) $\gamma=0.33$

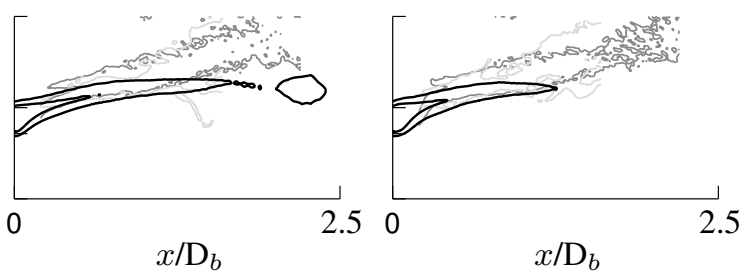

(e) $\gamma=0.85$
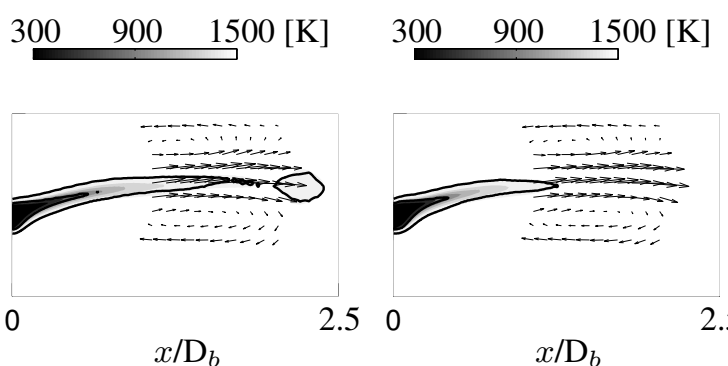

(h) $\gamma=0.85$

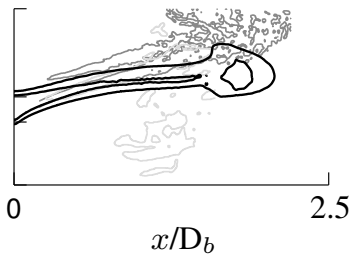

(c) $\gamma=0.66$

Fig. 12 Spatial extent of the reaction zone; (a)-(f) contour of the heat release rate, dark grey line: FSD data of Balachandran [5], light grey line: HRR data of Ayoola [4], black line: simulation, (g)-(i) filled grey contours, the black solid line, and arrows represent the predicted temperature field, the contour of the predicted heat release rate, and the velocity field, respectively.

At the extinction point the fluid temperature is close to the temperature of the burnt mixture and the radical mass fractions become insignificantly small, which suggests that the mixture approaches a fully burnt state. Beyond the extinction point the upstream part of the flame propagates back upstream towards the inlet of the combustion chamber while the downstream part extinguishes promptly further downstream. Fig. 10 shows that the axial velocity in the annular jet close to the combustor inlet (monitor P2) is at its minimum at $\gamma=0.80$. The minimum of the axial velocity is experienced with a spatially-dependent time delay further downstream along the annular jet and assumes a value which is less than the speed of flame propagation once it reaches the downstream end of the flame. As a result the downstream end of the flame propagates upstream relative to the fluid and the reaction zone exhibits local "necking" at the intersection of the elongated and the radially-widened part of the flame $\left(x / D_{b}=1.9\right.$ and $\left.r / D_{b}=0.9\right)$. The flame gradually propagates into opposite directions in the necking region which eventually detaches the elongated from the radially widened part of the flame. The influence of high stretching rates originating from the shear layers on the flame extinction is small since the flame extinction takes place well within the 
annular jet. It is noted that the phase at which the flame reaches its maximum spatial extent is not coincident with the phase at which the total heat release rate has its maximum. The maximum spatial extent of the reaction zone is reached at $\gamma=0.85$, whereas the maximum total heat release rate is at $\gamma=0.63$.

5.4 Measures for the heat release rate

Fig. 13 compares the measured bulk $\mathrm{OH}^{*}$ chemiluminescence with the simulated total heat release rate as a function of the phase $\gamma$. The time-averaged value of the calculated total heat release rate is $\left\langle Q_{t o t}\right\rangle_{t}=6.1 \mathrm{~kW}$. A noticeable feature in Fig. 13 is the distinct phase shift of $\Delta \gamma \in[0.2-0.056,0.2+0.056]$ between the $\mathrm{OH}^{*}$ chemiluminescence and $\left\langle Q_{t o t}\right\rangle_{\gamma}$. This observed phase shift is investigated in more detail below. In principle the phase shift can be investigated by estimating the mass fraction of the chemically excited $\mathrm{OH}^{*}$. The mass fraction is denoted by the square brackets. One way to compute $\left[\mathrm{OH}^{*}\right]$ quantitatively is to include a reaction mechanism for the formation and destruction of $\mathrm{OH}^{*}$, see for example $[16,18]$. However, Carl et al. [8] have shown that the reaction:

$$
\mathrm{CH}+\mathrm{O}_{2} \rightleftharpoons \mathrm{CO}+\mathrm{OH}^{*}
$$

plays an important part in the formation of $\mathrm{OH}^{*}$ in hydrocarbon combustion and that the emission intensity $\left[\mathrm{OH}^{*}\right]$ is directly proportional to the methylidyne radical $(\mathrm{CH})$ and molecular oxygen $\left(\mathrm{O}_{2}\right)$, namely $[\mathrm{CH}]\left[\mathrm{O}_{2}\right]$. Neither of these methods of obtaining $\left[\mathrm{OH}^{*}\right]$ were pursued in the present work. The additional reaction mechanism for $\mathrm{OH}^{*}$ would significantly increase the computational cost and $\mathrm{CH}$ is not included in the reduced reaction mechanism of Luo et al. [24]. Instead the less reliable ground-state $\mathrm{OH}$ mass fraction is used to investigate the observed phase shift. Justification for the use of $[\mathrm{OH}]$ is based upon the findings of Hall et al. [17], who demonstrated that the ratio $\mathrm{OH}_{\max } / \mathrm{OH}_{\max }^{*}$ remains approximately constant over a wide range of temperatures in a stoichiometric ethane-oxygen flame. The authors also found that both $\mathrm{OH}$ and $\mathrm{OH}^{*}$ tend to exhibit initial rapid formation around the same time for some of the investigated flames (fuel-rich and fuel-lean acetylene-oxygen and ethane-oxygen flames) leading to approximately the same ignition onset times. The $\mathrm{OH}^{*}$ chemiluminescence is a line-of-sight integration of the three-dimensional emission intensity and therefore the line-of-sight integration method of Ruder et al. [28] has been applied to the estimate the ground-state $\mathrm{OH}$ mass fraction field. The resulting bulk variation is also plotted in Fig. 13. It can be seen that both $\mathrm{OH}^{*}$ chemiluminescence and the estimated $[\mathrm{OH}]$ have approximately the same phase shift with regard to the total heat release rate, thus supporting the above finding. 


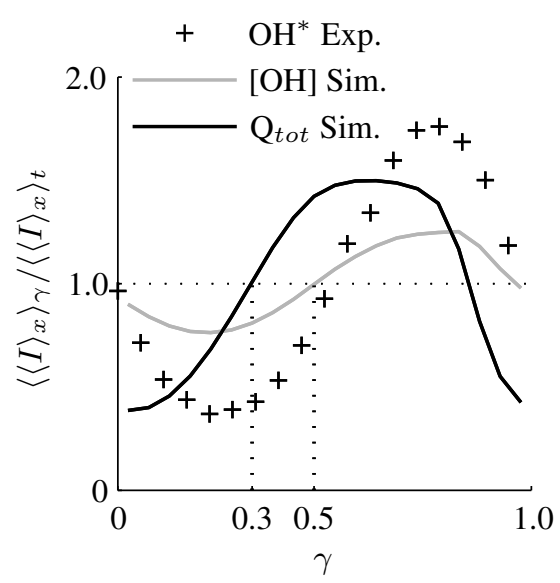

Fig. 13 Heat release rate as a function of $\gamma$; symbols: experimental data of Balachandran [5], solid lines: simulation.

Fig. 14 reveals that large fractions of the predicted $O H$ and measured $\mathrm{OH}^{*}$ chemiluminescence are found in the "post-reaction" zone where the mixture has not yet attained a state of strong chemical equilibrium. This same observation is made when $[\mathrm{OH}]$ is plotted in the median plane of the combustion chamber (not shown here) and implies that a time delay exists between the total heat release rate and the bulk formation of $O H$. However, the shape of the non-linear evolution of the total heat release rate compares well with the bulk $O H^{*}$ chemiluminescence.

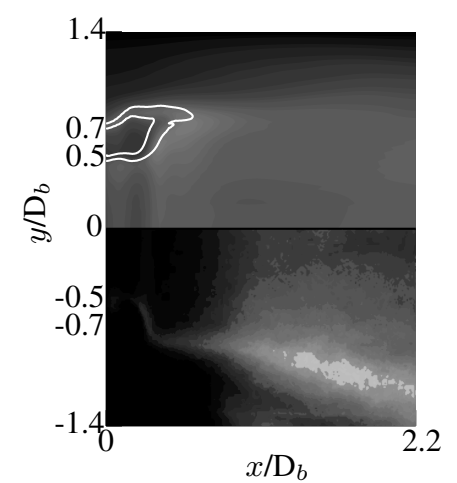

(a) $\gamma=0.0$

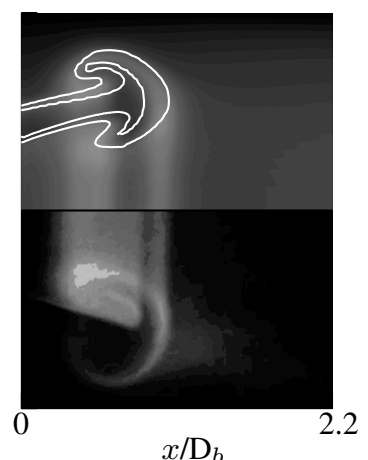

(b) $\gamma=0.33$

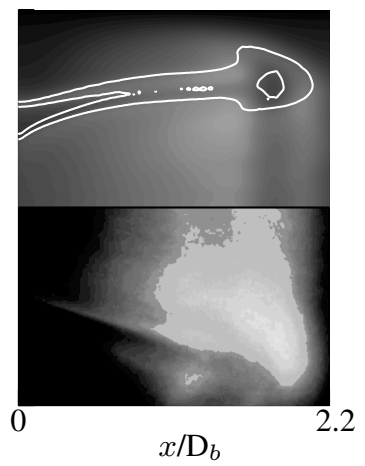

(c) $\gamma=0.66$

Fig. 14 Line-of-sight integration of the predicted $O H$ mass fraction field (upper half) and the $\mathrm{OH}^{*}$ chemiluminescence measurements of Balachandran [5] (lower half) in the median plane of the combustion chamber. The white contour represents the reaction zone.

The maximum values of the $\mathrm{OH}^{*}$ bulk intensity and $\left\langle Q_{t o t}\right\rangle_{\gamma}$ differ by approximately $15 \%$ due to different flame dynamics, ie the absence of the flame-wall interaction in the simulation. Nikolaou and Swaminathan [26] have recently investigated commonly used measures for the heat release rate by simulating flames in mixtures of methane $\left(\mathrm{CH}_{4}\right)$ and air. 
They found that for lean to stoichiometric conditions the reactions:

$$
\begin{aligned}
\mathrm{H}+\mathrm{CH}_{2} \mathrm{O} & \rightleftharpoons \mathrm{HCO}+\mathrm{H}_{2}, \\
\mathrm{O}+\mathrm{CH}_{4} & \rightleftharpoons \mathrm{OH}+\mathrm{CH}_{3},
\end{aligned}
$$

provide improved correlations with the heat release rate in comparison to the frequently used $[\mathrm{OH}]\left[\mathrm{CH}_{2} \mathrm{O}\right]$. The authors also identified the reactions

$$
\begin{aligned}
& \mathrm{H}+\mathrm{HO}_{2} \rightleftharpoons \mathrm{O}_{2}+\mathrm{H}_{2}, \\
& \mathrm{H}+\mathrm{HO}_{2} \rightleftharpoons \mathrm{O}+\mathrm{H}_{2} \mathrm{O}, \\
& \mathrm{H}+\mathrm{HO}_{2} \rightleftharpoons 2 \mathrm{OH} .
\end{aligned}
$$

as providing better estimates of the heat release rate in the laminar regime. The forward rates of (10), (11) and (12)-(14) are proportional to the products $[\mathrm{H}]\left[\mathrm{CH}_{2} \mathrm{O}\right],[\mathrm{O}]\left[\mathrm{CH}_{4}\right]$ and $[\mathrm{H}]\left[\mathrm{HO}_{2}\right]$, respectively. Gazi et al. [14] suggested that $\mathrm{CH}_{2} \mathrm{O}$ based measures for the heat release rate are valid when the major pathway of the fuel oxidation proceeds along reactions which involve the methyl radical $\left(\mathrm{CH}_{3}\right)$. The authors also identified the product of the mass fractions of molecular oxygen and the ketenyl radical (HCCO) as an adequate measure of the heat release rate in fuel-rich ethylene-air flames. All these measures are plotted in Fig. 15 as area-weighted averages of the simulated results in the median plane of the combustion chamber. It appears that $\left[\mathrm{O}_{2}\right][\mathrm{HCCO}]$ correlates best with the simulated total heat release rate in the flow configuration investigated here. Both the amplitude and phase of $\left\langle Q_{t o t}\right\rangle_{\gamma}$ are well reproduced using the product $\left[\mathrm{O}_{2}\right][\mathrm{HCCO}]$. On the other hand, $[\mathrm{OH}]\left[\mathrm{CH}_{2} \mathrm{O}\right],[\mathrm{H}]\left[\mathrm{CH}_{2} \mathrm{O}\right]$ and $[\mathrm{O}]\left[\mathrm{CH}_{4}\right]$ all suggest an approximately $30 \%$ higher maximum value of the total heat release rate at $\gamma \approx 0.7$. [H][HO2] additionally exhibits a kink at $\gamma \approx 0.7$.
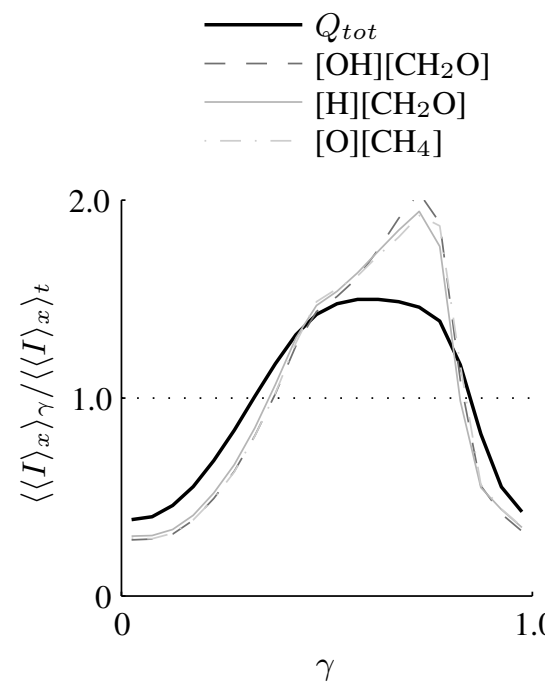
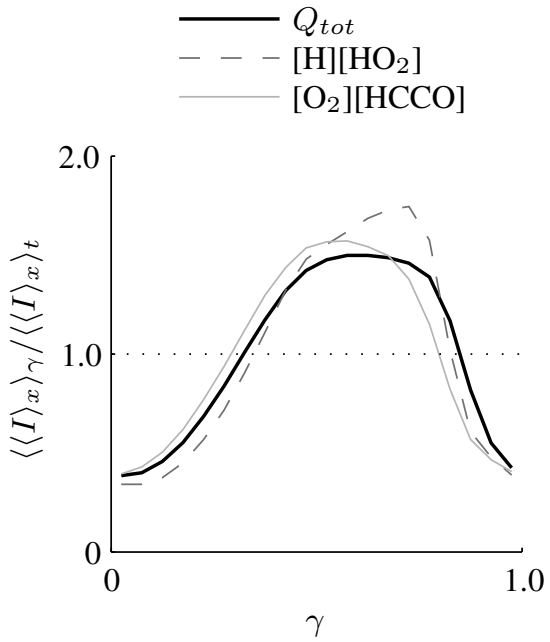

Fig. 15 Various integral measures to quantify the variation of the total heat release rate.

Reliable fluorescence imaging techniques already exist for the determination of the mole fraction of molecular oxygen $[12,15]$. Brock et al. [6] have developed laser-induced fluorescence spectroscopy of the ketenyl radical. This enables the experimentalist to determine 
the product $\left[\mathrm{O}_{2}\right][\mathrm{HCCO}]$ and test its application in similar flame configurations. It has to be acknowledged that the findings may not apply to other flame configurations since the major chemical pathway for the fuel oxidation and hence the chemical reactions correlating well with the overall heat release rate depend on many parameters, including the type of fuel and the equivalence ratio.

\section{Summary and conclusions}

The present paper reports the results of the simulation of an oscillating flame in a bluff-body stabilised combustor with the LES-PDF approach. The PDF transport equations are solved using the Eulerian stochastic field method and the chemical reactions are represented by a detailed but reduced reaction mechanism. It is first shown that the LES model is capable of reproducing the first and second moments of the measured velocity field of the nonoscillating and oscillating cold flows with good accuracy. The response of the flame to the imposed sinusoidal excitation of the mass flow rate is then studied and goof agreement with measurements is found. The non-linear evolution of the total heat release rate is especially well captured. A time lag of approximately $20 \%$ of the time period associated with the forcing frequency between the measured $\mathrm{OH}^{*}$ chemiluminescence and the predicted total release rate was identified and examined. This time lag can be attributed to the finding that the bulk of the $\mathrm{OH}$ mass fraction is only formed in the post-reaction zone where the contribution to the total heat release rate is low. It remains a subject for future work to investigate whether or not the time lag depends on the frequency of the oscillation. Various alternative measures to quantify the variation of the total heat release rate were investigated. In the present study of an oscillating lean ethylene-air flame it is found that $\mathrm{CH}_{2} \mathrm{O}$ based measures do not appropriately represent the variation of total heat release rate. Instead, the product of molecular oxygen and the ketenyl radical $\left[\mathrm{O}_{2}\right][\mathrm{HCCO}]$ shows the best agreement with the total heat release rate. Laser diagnostics for the determination of $\left[\mathrm{O}_{2}\right][\mathrm{HCCO}]$ could help validate the findings of the present work.

Acknowledgements The authors greatly acknowledge the funding provided by Siemens Industrial Turbomachinery Ltd.

\section{References}

1. Ahmed, S. F., Balachandran, R., Marchione, T., Mastorakos, E.: Spark ignition of turbulent nonpremixed bluff-body flames. Combust. Flame 151, 366-385 (2007)

2. Armitage, C. A., Balachandran, R., Mastorakos E., Cant, R. S.: Investigation of the nonlinear response of turbulent premixed flames to imposed inlet velocity oscillations. Combust. Flame 146, 419-436 (2006)

3. Ayache, S., Dawson, J. R., Triantafyllidis, A., Balachandran, R., Mastorakos, E.: Experiments and LargeEddy Simulations of acoustically forced bluff-body flows. Int. J. Heat Fluid Flow 31, 754-766 (2010)

4. Ayoola, B. O.: Laser-based measurement of heat release rate and temperature in turbulent premixed flames. Ph.D. thesis Cambridge University (2005)

5. Balachandran, R.: Experimental Investigation of the Response of Turbulent Premixed Flames to Acoustic Oscillations. Ph.D. thesis Cambridge University (2005)

6. Brock, L. R., Mischler, B., Rohlfing, E. A.: Laser-induced fluorescence spectroscopy of the ketenyl radical. J. Chem. Phys. 107, 665-668 (1997)

7. Bulat, G., Jones, W. P., Marquis, A. J.: Large Eddy Simulation of an industrial gas-turbine combustion chamber using the sub-grid PDF method. P. Combust. Inst. 34, 3155-3164 (2013)

8. Carl, S. A., Van Poppel, M., Peeters, J.: Identification of the $\mathrm{CH}+\mathrm{O}_{2} \rightarrow \mathrm{OH}(\mathrm{A})+\mathrm{CO}$ Reaction as the Source of $\mathrm{OH}(\mathrm{A}-\mathrm{X})$ Chemiluminescence in $\mathrm{C}_{2} \mathrm{H}_{2} / \mathrm{O} / \mathrm{H} / \mathrm{O}_{2}$ Atomic Flames and Determination of Its Absolute Rate Constant over the Range T = 296 to 511 K. J. Phys. Chem. 107, 11001-11007 (2003) 
9. di Mare, L., Klein, M., Jones, W. P., Janicka, J.: Synthetic turbulence inflow conditions for large-eddy simulation. Phys. Fluids. 18, (025107)1-11 (2006)

10. da Silva, C. B.: The behaviour of sub-grid-scale models near the turbulent/non-turbulent interface in jets. Phys. Fluids 21, (081702)1-4 (2009)

11. da Silva, C. B. Balarac, G., Métais, O.: Transition in high velocity ratio coaxial jets analysed from direct numerical simulations. J. Turbul. 4, 1-18 (2003).

12. Di Rosa, M. D., Klavuhn, K. G., Hanson, R. K.: LIF Spectroscopy of NO and $\mathrm{O}_{2}$ in High-Pressure Flames. Combust. Sci. and Tech. 118, 257-283 (1996).

13. Gao, F., O’Brien, E. E.: A large-eddy simulation scheme for turbulent reacting flows. Phys. Fluids 5, 1282-1284 (1993)

14. Gazi, A., Vourliotakis, G., Skevis, G., Founti, M. A.: Assessment of Chemical Markers for Heat-Release Rate Correlations in Laminar Premixed Flames. Combust. Sci. Technol. 185, 1482-1508 (2013)

15. Goldman, A., Rahinov, I., Cheskis, S.: Molecular oxygen detection in low pressure flames using cavity ring-down spectroscopy. Appl. Phys. B 82, 659-663 (2006)

16. Haber, L. C., Vandsburger, U.: A Global Reaction Model for $\mathrm{OH}^{*}$ Chemiluminescence applied to a Laminar Flat-Flame Burner. Combust. Sci. and Tech. 175, 1859-1891 (2003)

17. Hall, J. M., Rickard, M. J. A., Petersen, E. L.: Comparison of Characteristic Time Diagnostics for Ignition and Oxidation of Fuel/Oxidiser Mixtures behind Reflected Shock Waves. Combust. Sci. Technol. 177, 455483 (2005)

18. Hidaka, Y., Takahashi, S., Kawano, H., Suga, M., Gardiner, W. C. Jr.: Shock-Tube Measurement of the Rate Constant for Excited $\mathrm{OH}\left(\mathrm{A}^{2} \Sigma^{+}\right)$Formation in the Hydrogen-Oxygen Reaction. J. Phys. Chem. 86, 1429-1433 (1982)

19. Jones, W. P., Prasad, V. N.: Large Eddy Simulation of the Sandia Flame Series (D-F) using the Eulerian stochastic field method. Combust. Flame 157, 1621-1636 (2010)

20. Sabel'nikov, V., Soulard, O.: Rapidly decorrelating velocity-field model as a tool for solving one-point Fokker-Planck equations for probability density functions of turbulent reactive scalars. Phys. Rev. E 72, (016301)1-22 (2005)

21. Jones, W. P., Marquis A. J., Prasad, V. N.: LES of a turbulent premixed swirl burner using the Eulerian stochastic field method. Combust. Flame 159, 3079-3095 (2012)

22. Jones, W. P., Marquis, A. J., Noh, D.: LES of a methanol spray flame with a stochastic sub-grid model. P. Combust. Inst. 35, 1685-1691 (2015)

23. Klein, M., Sadiki, A., Janicka, J.: A digital filter based generation of inflow data for spatially developing direct numerical or large eddy simulations. J. Comput. Phys. 186, 652-665 (2003)

24. Luo, Z., Yoo, C. S., Richardson, E. S., Chen, J. H., Law, C. K., Lu, T.: Chemical explosive mode analysis for a turbulent lifted ethylene jet flame in highly-heated co-flow. Combust. Flame 159, 265-274 (2012)

25. Matalon, M.: Intrinsic Flame Instabilities in Premixed and Nonpremixed Combustion. Annu. Rev. Fluid Mech. 39, 163-191 (2007)

26. Nikolaou, Z. M., Swaminathan, N.: Heat release rate markers for premixed combustion. Combust. Flame 161, 3073-3084 (2014)

27. Piomelli, U., Liu, J.: Large-eddy simulation of rotating channel flows using a localised dynamic model. Phys. Fluids. 7, 839-848 (1995)

28. Ruder, H., Ertl, T., Geyer, F., Herold, H., Kraus, U.: Line-of-sight integration: A powerful tool for visualisation of three-dimensional scalar fields. Comput. Graph. 13, 223-228 (1989)

29. Triantafyllidis, A., Mastorakos, E., Eggels, R. L. G. M.: Large Eddy Simulations of forced ignition of a non-premixed bluff-body methane flame with Conditional Moment Closure. Combust. Flame 156, 2328$2345(2009)$ 